\title{
Compound Nevus Occurring Near External Auditory Canal: Successful Treatment by $\mathrm{CO}_{2}$ Laser Abrasion
}

\author{
Won-Serk Kim ${ }^{1}$ and Kye Hoon Park ${ }^{2}$ \\ ${ }^{1}$ Department of Dermatology, Kangbuk Samsung Hospital, Sungkyunkwan University School of Medicine, Seoul, \\ ${ }^{2}$ Department of Otorhinolaryngology-Head and Neck Surgery, Cheonan Hospital, Soonchunhyang University College of Medicine, \\ Cheonan, Korea
}

Received March 5, 2013

Revised March 21, 2013

Accepted March 24, 2013

\author{
Address for correspondence \\ Kye Hoon Park, MD \\ Department of Otorhinolaryngology- \\ Head and Neck Surgery, \\ Cheonan Hospital, \\ Soonchunhyang University \\ College of Medicine, \\ 31 Suncheonhyang 6-gil, \\ Dongnam-gu, Cheonan 330-721, \\ Korea \\ Tel +82-41-570-3801 \\ Fax +82-41-579-9022 \\ E-mail earpark@gmail.com
}

Melanocytic nevus is common pigmented skin tumor, but it has rare occurrence near the external auditory canal. A skin graft is required if a wide surgical excision is performed for the nevi occurring near the external auditory canal. $\mathrm{A} \mathrm{CO}_{2}$ laser was considered to be a good alternative for the removal of melanocytic nevus in this area. We herein report a case of compound nevus occurring near the external auditory canal that was successfully treated by $\mathrm{CO}_{2}$ laser abrasion.

Korean J Audiol 2013;17:30-31

KEY WORDS: Melanocytic nevus · External auditory canal · Laser.

\section{Introduction}

Melanocytic nevus can occur at any site in the human body, but its development near the external auditory canal is extremely rare, where surgical removal is difficult. Considering the thin and taut ear skin, poor surgical field, and difficult postoperation wound care, we considered a $\mathrm{CO}_{2}$ laser to be a good alternative for the removal of melanocytic nevus in this area. We herein report a case of compound nevus occurring near the external auditory canal that was successfully treated by $\mathrm{CO}_{2}$ laser abrasion.

\section{Case Report}

A 47-year-old Korean man presented with a 1-year history of a well-demarcated $1 \times 1 \mathrm{~cm}$, dark-brown to black papillomatous plaque in his right cavum conchae (Fig. 1). He didn't complain of any pain, itching, intermittent oozing, or hearing disturbance. On otoscopic examination, his right tympanic membrane and external auditory canal were normal. He was healthy and had no history of skin cancer or other skin problem. A skin biopsy specimen showed numerous nests and clumps of melanocytic nevus cells in the upper dermis and dermoepidermal junction (Fig. 2). The lesion was treated with $\mathrm{CO}_{2}$ laser (Model 1020C 20WA, Sharplan Lasers Inc., Migdal Haemek, Israel) abrasion at 1.0 watt in the super-pulsed mode. Only topical antibiotic ointment without coverage was applied for post-laser wound care for 1 week. The patient showed no recurrence during the 6-month follow-up period (Fig. 3).

\section{Discussion}

The common acquired melanocytic nevus is a benign neoplastic proliferation of melanocytes. Histologically it is diagnosed by the presence of nevus cells that are arranged in clusters or nests, and is classified into junctional, compound, and intradermal nevi, in which the nevus cells are confined to the epidermis, epidermis and dermis, and dermis respectively. In addition to the histological classification, five clinical types of lesion can be recognized: 1) flat, 2) slightly elevated often 


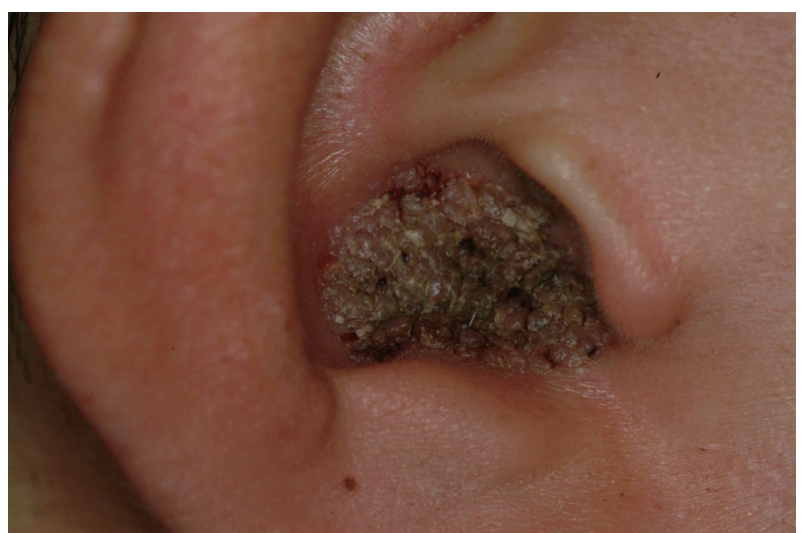

Fig. 1. A well-demarcated $1 \times 1 \mathrm{~cm}$, dark-brown to black papillomatous plaque near the external auditory canal of right ear.

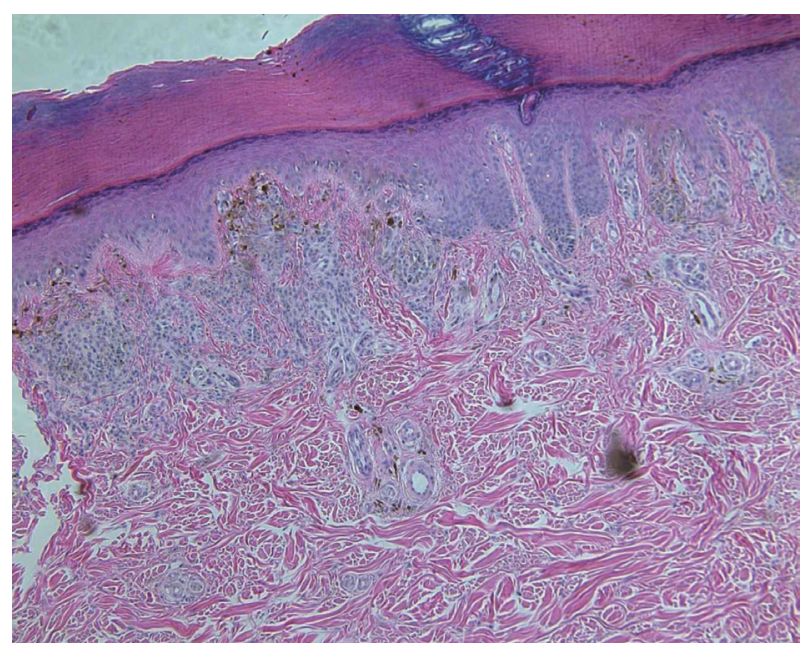

Fig. 2. Numerous nests and clumps of melanocytic nevus cells in the upper dermis and dermoepidermal junction ( $\times 100, H \& E)$.

with raised centers and flat peripheries, 3) papillomatous, 4) dome-shaped, and 5) pedunculated. ${ }^{1)}$ In most cases, slightly elevated lesions represent compound nevi, while papillomatous, dome-shaped, and pedunculated lesions represent intradermal nevi. Friedmann ${ }^{2)}$ reported the first case of melanocytic nevus in the skin of the external auditory canal and two cases of melanocytic nevi presenting as papillomatous lesions that were revealed as intradermal nevi have been described in the English literature. ${ }^{3,4)}$ In our case, the lesion was a papillomatous plaque similar to the previously reported cases, but it turned out to be a compound nevus.

Melanocytic nevi occurring in the auricular region, like those occurring in the acral, flexural, or genital area, may exhibit some of the histologic features commonly found in melanoma such as pagetoid spread of melanocytes, cytologic atypia, and asymmetric growth pattern. ${ }^{5}$ Therefore, careful histologic interpretation of these rare lesions is recommended. The differ-

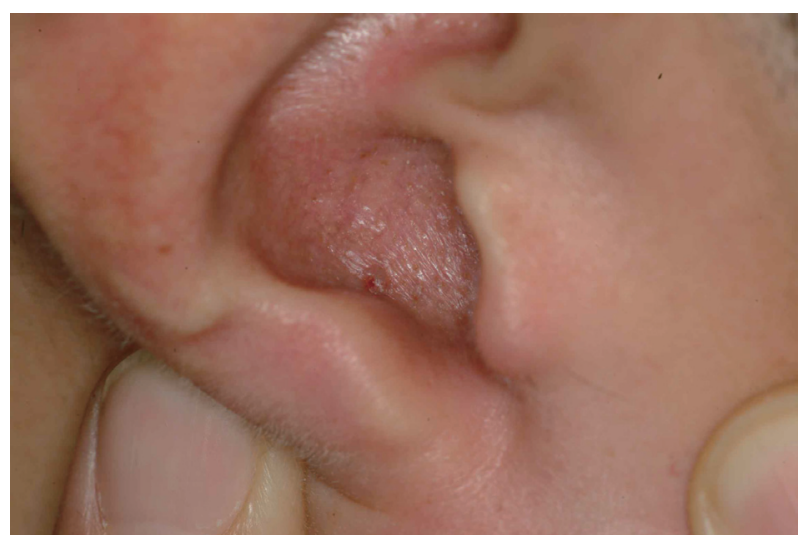

Fig. 3. Clearance of the skin lesion (after the 6-month of a single session of laser abrasion).

ential diagnosis should include viral wart, seborrheic keratosis, foreign body granuloma, and a variety of benign and malignant neoplasms.

The $\mathrm{CO}_{2}$ laser has been widely used for the removal of various skin tumors, including melanocytic nevus. However, it was usually effective only in small superficially located tumors with surgical excision being recommended for large or deeply seated tumors. As mentioned above, the wide surgical excision of nevi occurring near the external auditory canal is difficult, and hence we attempted $\mathrm{CO}_{2}$ laser abrasion despite the large size in the case presented here. We had expected several treatments to be necessary, but found that a single session of treatment was sufficient for clearance.

To the best of our knowledge, this is the first case of a compound papillomatous nevus occurring near the external ear canal. We consider that compound nevus should be included in the differential diagnosis of papillomatous skin lesion occurring near the external auditory canal and that $\mathrm{CO}_{2}$ laser abrasion can be a good choice for its treatment.

\section{REFERENCES}

1) Elder D, Elenitsas R, Murphy G, Xu X. Benign pigmented lesions and malignant melanoma. In: Elder D, editor. Lever's histopathology of the skin. 9th ed. Philadelphia: Lippincott-Raven;2005. p.725-31.

2) Friedmann J. Squamous epithelial neoplasms. In: Friedmann J, editor. Pathology of the ear. 1st ed. Oxford Blackwell Scientific Publications;1974. p.156.

3) Kazikdas KC, Onal K, Kuehnel TS, Ozturk T. An intradermal nevus of the external auditory meatus. Eur Arch Otorhinolaryngol 2006; 263:253-5.

4) Alves RV, Brandão FH, Aquino JE, Carvalho MR, Giancoli SM, Younes EA. Intradermal melanocytic nevus of the external auditory canal. Braz J Otorhinolaryngol 2005;71:104-6.

5) Saad AG, Patel S, Mutasim DF. Melanocytic nevi of the auricular region: histologic characteristics and diagnostic difficulties. Am J Dermatopathol 2005;27:111-5. 